\title{
ITER GLOBAL STABILITY LIMITS
}

\author{
J. T. HOGAN and N. A. UCKAN \\ Fusion Energy Division \\ Oak Ridge National Laboratory \\ P.O. Box 2009 \\ Oak Ridge, TN, 37830-8072
}

\section{ABSTRACT}

The MHD stability limits to the ITER operational space have been examined with the PEST ideal stability code. Coristraints on ITER operation have been examined for the nominal operational scenarios and for possible design variants. Rather than rely on evaluation of a relatively small number of sample cases, the approach has been to construct an approximation to the overall operational space, and to compare this with the observed limits in high- $\beta$ tokamaks. An extensive database with $\sim 20,000$ stability results has been compiled for use by the ITER design team. Results from these studies show that the design values of the Troyon factor (g 2.5 for ignition studies, and g 3 for the technology phise) which are based on present experiments, are also expected to be attainable for ITER conditions, for which the configuration and wall-stabilisation environment differ from those in present experiments. Strongly peaked pressure profiles lead to degraded high- $\beta$ performance. Values of $g \sim 4$ are found for higher safety factor $\left(q_{\Psi} \geq 4\right)$ than that of the present design $\left(q_{\Psi} \sim 3\right)$. Profiles with $q(0)<1$ are shown to give $g \sim 2.5$, if the current density piofile provides optimum shedr. The overall operational spaces are presented for $g-q_{\psi}, q_{\psi}$ $l_{i}, q-\alpha_{p}$ and $l_{i}-q_{\psi}$.

\section{MODEL / DATABASE}

\section{PEST Model}

The calculacions reported here are carried out with the PEST system of codes. The equilibriun: calculations use the flix coordinate inverse equilibrium solver of Delucia, Jardin, and Todd [1] as a fixed boundary code to solve the iwo dimensional axisymmetric Grad-Shafranov equation in toroidal geometry, while the PEST 2.4 code [2] (provided by J. Manickam of PPPL) is used for low-n stability. The PEST code sy'stem has also been used to evaluate ballooning stability. For large-scale surveys 20 equally spaced flux surfaces were studied, so that small zones of ballooning instability can be missed. Stability of the $n=1$ mode is determined with the PEST 2.4 code with the conducting

\footnotetext{
*hesearch sporsored by the office of Fusion Enerar, U.S. Department of EnErEy, under cortract DE-4C05-840R21400 with Wartin Marietta Enerey Evsiers, Inc. 
wall removed to $i_{\text {..inity }}$. As to the numerical details the PEST mapper uses 201 flux surfaces, 128 angle points, with poloidal mode selections of $m=-5$ to 15 .

\section{Equilibrium parameter ranges}

Calculations have been made for several ITER variants, so the system parameters have the following range :

$\begin{array}{llll}\text { Aspect ratio } & \mathrm{A}=2.6 \rightarrow 3.1 & \text { Elongation } & \kappa=1.8 \rightarrow 2.2 \\ \text { Triangularity } & \delta=0.2 \rightarrow 0.6 & \text { Edge safety factor } & \mathrm{q}_{\psi}=2.5 \rightarrow 7 . \\ \text { Central } \mathrm{q} & \mathrm{q}(0)=0.85 \rightarrow 2.5 & <\beta> & \text { scanned continuously }\end{array}$

These values correspond to ITER ignition studies and technology variants. The exact values are described for each case as it is discussed.

\section{Configuration and profiles}

The shape of the outer flux surface is given by $R=R_{0}+a \cos (\theta+\delta \sin (\theta))$ and $Z=\kappa a$ $\sin (\theta)$ where $R_{0}$ is the major radius, $a$ is the minor radius $\left(A=R_{0} / a\right), k$ is the elongation, and $\delta$ is the triangularity. The maximum value of $z\left(z^{\max }=\kappa a\right)$ occurs at $R=R_{0}$-a $\sin (\delta)$. The forms assumed for the pressure and safety factor profiles are:

$$
\begin{gathered}
p=p_{0}\left(1-\psi^{\alpha_{0}}\right)^{\tau}+p_{1} \psi^{3}(1-\psi)^{1.75} \\
q=q_{0}+q_{1} \psi^{\alpha_{1}}+q_{2} \psi^{\alpha_{2}}
\end{gathered}
$$

As to the pressure profile parameters, $p_{0}$ has been varied to scan $\beta, p_{1}=0$ and $1, \alpha_{0}$ was varied from 0.75 to 2.0 , and $\tau$ from 0.8 to 2.4. For cases in which $p_{1}=0$. and $\alpha_{0}=1$, the pressure profile is of the form $p=p_{0}(1-\psi)^{\alpha}$. For this case, the pressure profile peaking factor $p_{0} /<p>\sim 1+\alpha_{p}$. The parameter $\alpha_{p}$ is used as a measure of profile peaking for some cases, and is defined as $\alpha_{p} \equiv p_{0}<<p>-1$. For the q profile parameters: $q_{\psi}$, which is equal to $q_{0}+q_{1}+q_{2}$, varied from 2.5 to $7, q_{0}$ varied from 0.85 to $2.5, q_{1}$ was varied from 0.95 to $2.55, q_{2}$ took values from 0 to 2 , while $\alpha_{1}=2.5$ and 6.0 , and $\alpha_{2}=2$.

\section{Database}

Even though ideal MHD stabiiity is a relatively well posed and understood problem, the parameter space required to specify stability is large. The geometry and configuration requires the specification of the aspect ratio, elongation and triangularity (for double null configurations without indentation), the $q$ profile requires $q_{0}, q_{\psi}$ and at least one 
additional parameter (to determine whether the $q$ profile is concave or convex). [Note that five q-profile parameters are used in the present analysis]. The pressure profile, assuming $\mathrm{p}_{\text {edge }}=0$ requires at least $\mathrm{p}(0)$ and $\langle\mathrm{p}\rangle$. Addition of the mode number and position of the conducting wall brings the minimum number of parameters to 10 . Thus, even if only 3 values are computed per variable choice, $3^{10}(\sim 60,000)$ results must be obtained and sorted, while a more realistic choice of 5 values requires $5^{10}$ combinations $\left(\sim 10^{7}\right.$ results, requiring $~ 37$ CRAY-1 years). Thus, careful choice of calculations must be made, and attention paid to organising the results. The equilibrium and stability results have therefore been compiled in a database for use by the ITER design team. Figures 1 (a)-(d) show the cases evaluated for stability for the results presented here. Fig. 1(a) shows the $g-q_{\psi}$ space, Fig. 1(b) shows the $g-l_{i}$ space, Fig. 1(c) gives the $l_{i}-q_{\psi}$ space and Fig. 1(d) the $g-p /\langle p\rangle$ space. The specific form of internal inductance used is:

$$
\mathrm{l}_{\mathrm{i}}(3) \equiv 2<\mathrm{B}_{\mathrm{pol}}{ }^{2}>\mathrm{V} / \mu_{0}{ }^{2} \mathrm{I}_{\mathrm{p}}{ }^{2} \mathrm{R}_{\text {axis }}
$$

\section{OPERATING SPACE AND HIGH-q LIMITS}

\section{Rationale}

Recent high- $\beta$ results obtained on the DIII-D tokarnak have shown that the stable operation space can be expanded for $q_{\psi}>4$. Values g 4-5 were found experimentally [3]. This parameter regime is of potential interest for ITER, especially for current driven operation, where high- $\mathrm{q}_{\psi}$ may be desirable [4]. The DIII-D results cannot be directly adapted for ITER because the the poloidal field system in DIII-D introduces a stabilising effect which may not be present in steady-state ITER operation. Thus, calculations to determine the attainable $\mathrm{g}$ for ITER in the regime $4<\mathrm{q}_{\psi}<7$ have been performed.

\section{Resulis}

Figure 2 shows the predicted overall stable operational space for $g$ vs $\mathrm{q}_{\psi}$. A maximum Troyon coefficient $g \sim 4$ is obtained for $\mathrm{q}_{\psi} \sim 4$ and is found to be relatively constant for $\mathrm{q}_{\psi}>4$. Operation with $\mathrm{q}_{\psi}<3$ is predicted to lead to a diminution of stable $\mathrm{g}$. This is in contradiction with present experimental results, for which the maximum $g$ values are obtained for $\mathrm{q}_{\psi} \sim 2$. The reference design value for ITER ignition studies is $g=2.5$, based on the experimental database of discharges which have maintained this value for at least $1 \mathrm{sec}$, and for which the probability of $\beta$-related disruption or collapse is less than $10 \%$. Figure 3 shows the predicted stable $\mathrm{g} \mathrm{l}_{\mathrm{i}}(3)$ space, indicating a broad range of cases with $\mathrm{l}_{\mathrm{i}}(3) \sim 0.65$, 
the nominal ITER value. Figure 4 shows the calculated ITER $l_{i}(3)-q_{\psi}$ space for $g>2$. The region with $\mathrm{l}_{\mathrm{i}}(3)<0.6$ shows the 'ravine'-like behavior due to edge modes which are sensitive ts the distribution of current density. (Such behavior is seen in the operational regime for start-up in large tokamaks such as JET). A broad operational space for $3<q_{\Psi}<4$ is found to be accessible at the ITER reference value $\mathrm{l}_{\mathrm{i}}(3)=0.65$ Finally, in Figure 5 , the degradation in beta limits due to profile peaking is shown ( $g-p_{0} /<p>$ ). Values near the ITER requirement $(g \sim 2.5)$ are found for moderately peaked profiles, $\alpha_{p} \sim 1.5$.

\section{LIMITS WITH $q(0)<1$}

\section{Rationale}

While diagnostics in present experiments are generally inadequate to measure the q.profile in high- $\beta$ cases, the presence of sawtooth phenomena suggests that $q(0) \sim 1$ or less. Ideal MHD analysis shows that stability is sensitively deperident on the shape of the a profile in this case. Results are found which can differ strongly from the optimised profiles, for which $q(0) \sim 1+\varepsilon$ is chosen to avoid local modes near the axis. An analysis has thus been carried out to evaluate the ITER stability limits for profiles with $q(0)<1$.

\section{Results}

Figure 6 shows the stable cases for $q(0)=0.85$ as a function of internal inductance $1_{i}(3)$. The ITER ignition studies value $(g=2.5)$ is attained for $l_{i}(3)=0.65$, which is a nominal value for the reference configuration. Operation at higher values of the Troyon factor is facilitated by flattening the pressure profile in the center. This may be a self-consistent result of sawtooth flattening as well. Figure 7 shows the dependence of $g$ on the pressure prosile peaking factor, $\alpha_{p}$, where $p=p_{0}(1-\psi)^{\alpha_{p}}$ for cases with $q(0)=0.85$ The maximum stable $g$ decreases from 2.5 to $\sim 1.6$ as the peaking factor increases from 1 to 2.5 .

\section{CONCLUSIONS}

The ITER guidelines are based primarily on the demonstrated performance of high-beta experiments such as DIII-D, JET, ASDEX, JFT-2M, and PBX/M. These results cannot be applied directly to ITER, since the proposed ITER configuration and wall stabilisation envi--nment differ from these experiments. However, relatively good agreement between the Troyor ideal MHD model and experiments allows us to calculate the expected 
operational limits in TTER. These calculations show that the required high-beta performance in ITER can be attained for reasonable profiles with the configuration chosen. Questions remain as to the appropriate limit for non-symmetric configurations (i.e., single null divertors), scenarios with a large fast-ion population (kinetic effects) and the effect of $m=1$ (sawtooth-related) modes on stability.

\section{REFERENCES}

1. J. DeLucia, S. Jardin, A. Todd, Princeton Plasma Physics Laboratory Report PPPL1564, (1979)

2. R. Grimm, R. Dewar, J. Manickam, Jour. of Computat. Physics, 49 (1983) 94.

3. E.J. Strait and the DIII-D Physics Group, ITER Workshop on Beta Limits and Profiles, IPP-Garching, March 1990.

4. L.J. Perkins and J. Galambos, ITER-IL-SA-1-0-21, July 1990.

\section{FIGURE CAPTIONS}

Figure 1. The equilibria which have been examined for stability : Note: many of these cases are subsequently found to be unstable.
(a) $g$ (Troyon coefficient) $v s q_{\psi}$ (surface safety factor) space
(b) $g$ (Troyon coefficient) vs $l_{i}(3)$ (internal inductance) space
(c) $l_{i}(3)-q_{\psi}$ space
(d) g vs $\mathrm{p}_{0} /<\mathrm{p}>$ (pressure profile peaking factor) space

Figure 2 Predicted $g-q_{\psi}$ operational space : stable cases (for $n=1$, wall at infinity and ballooning).

Figure 3 Predicted g- $l_{i}(3)$ operational space: stable cases (for $n=1$, wall at infinity and ballooning).

Figure 4 Predicted $l_{i}(3)-q_{\psi}$ operational space: stable cases (for $n=1$, wall at infinity and ballooning).

Figure 5 Predicted $g-p_{0} /<p>$ operational space: stable cases (for $n=1$, wall at infinity and ballooning).

Figure 6 Predicted $g-l_{i}(3)$. operational space: stable cases (for $n=1$, wall at infinity and ballooning) for $q(0)=0.85$ 
Figure 7 Predicted g- $p_{\sigma} /<p>$ operational space: stable cases (for $n=1$, wall at infinity and ballooning) for $\mathrm{q}(0)=0.85$

\section{DISCLAIMER}

This report was prepared as an account of work sporisored by an agency of the United States Government. Neither the United States Government nor any agency thereof, nor any of their employees, makes any warranty, express or implied, or assumes any legal liability or responsibility for the accuracy, completeness, or usefulness of any information, apparatus, product, or process disclosed, or represents that its use would not infringe privately owned rights. Reference herein to any specific commercial product, process, or service by trade name, trademark, manufacturer, or otherwise does not necessarily constitute or imply its endorsement, recommondation, or favoring by the United States Government or any agency thereof. The views and opinions of authors expressed herein do not necessarily state or reflect those of the United States Government or any agency thereof. 

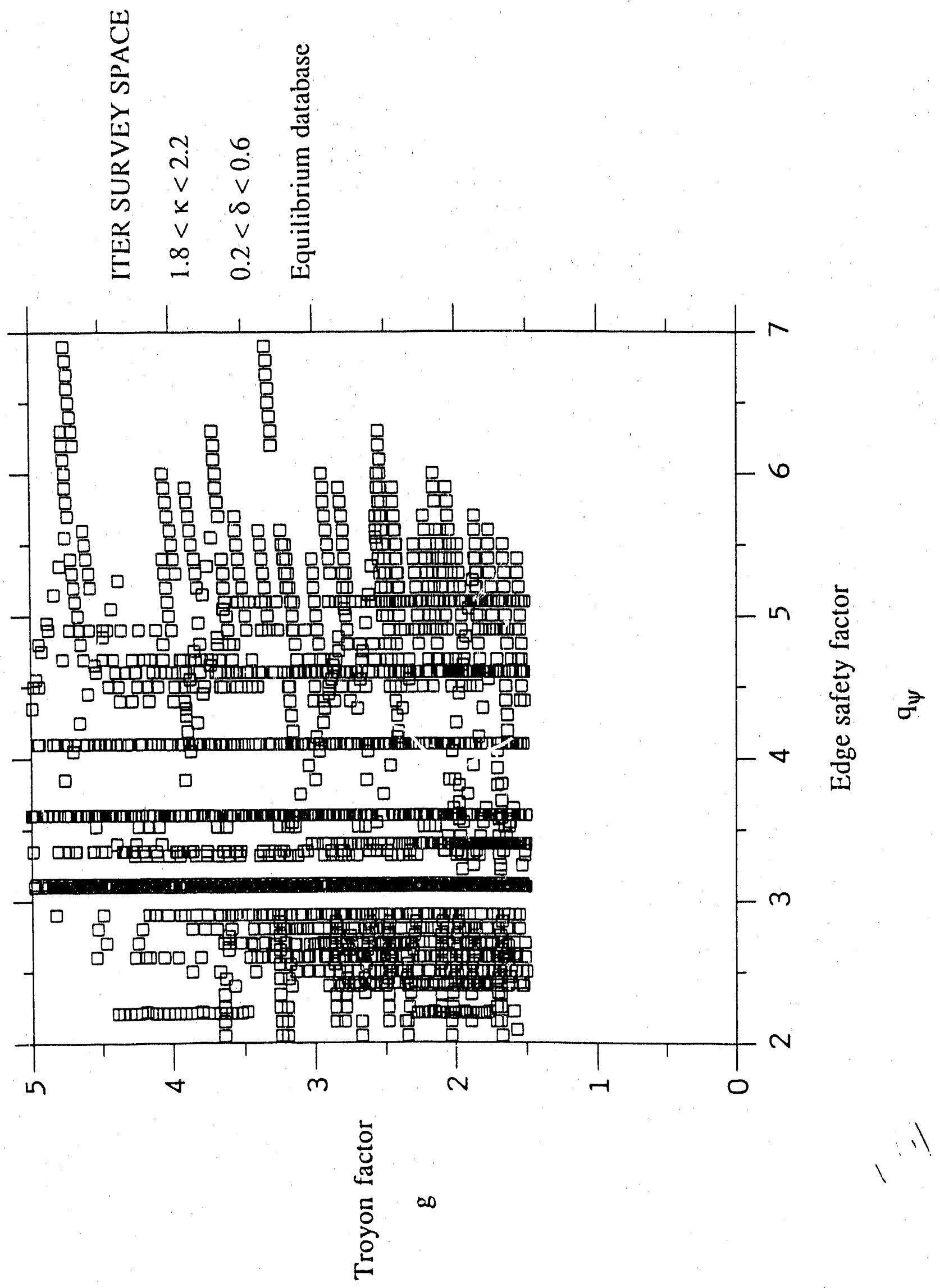

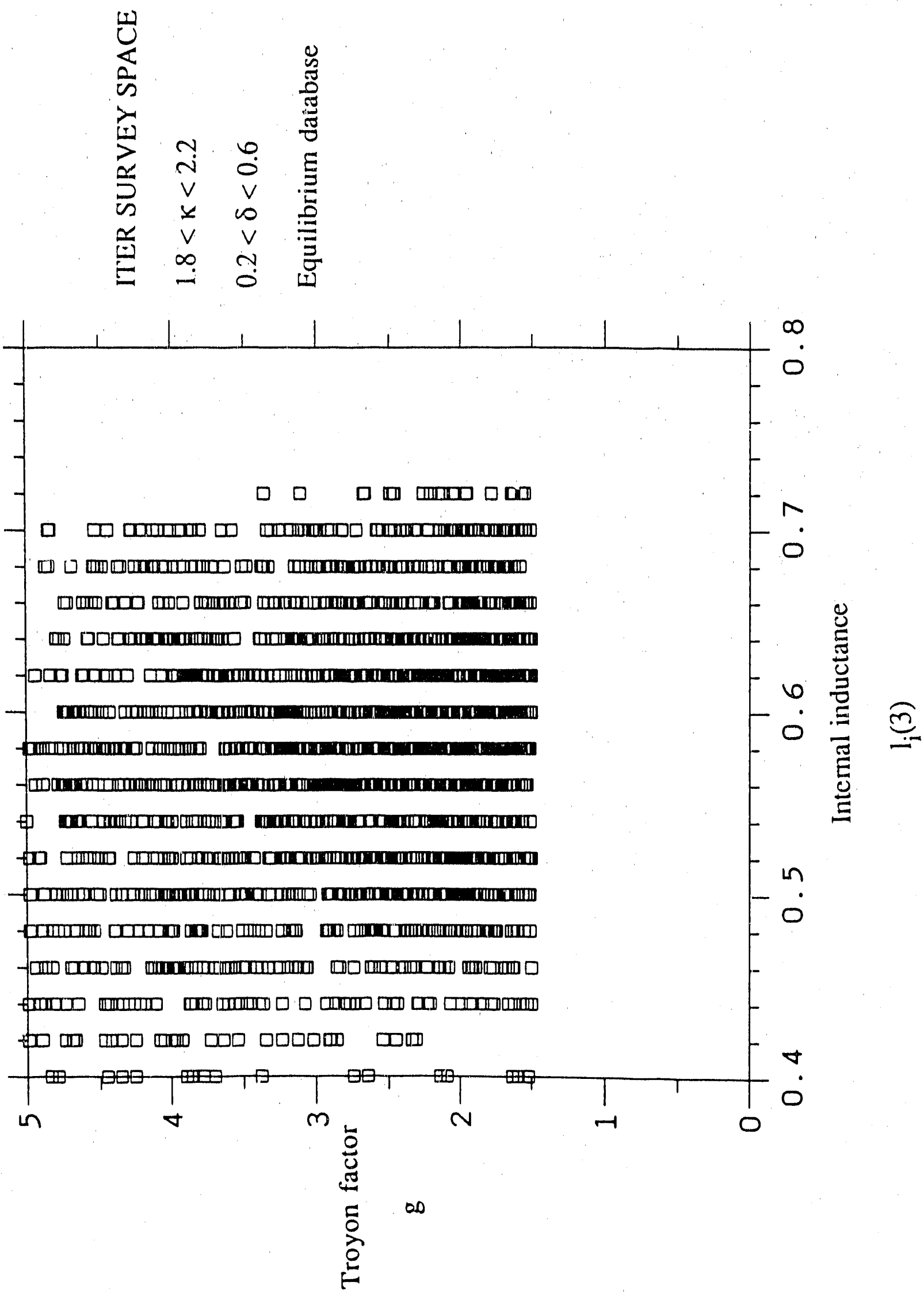

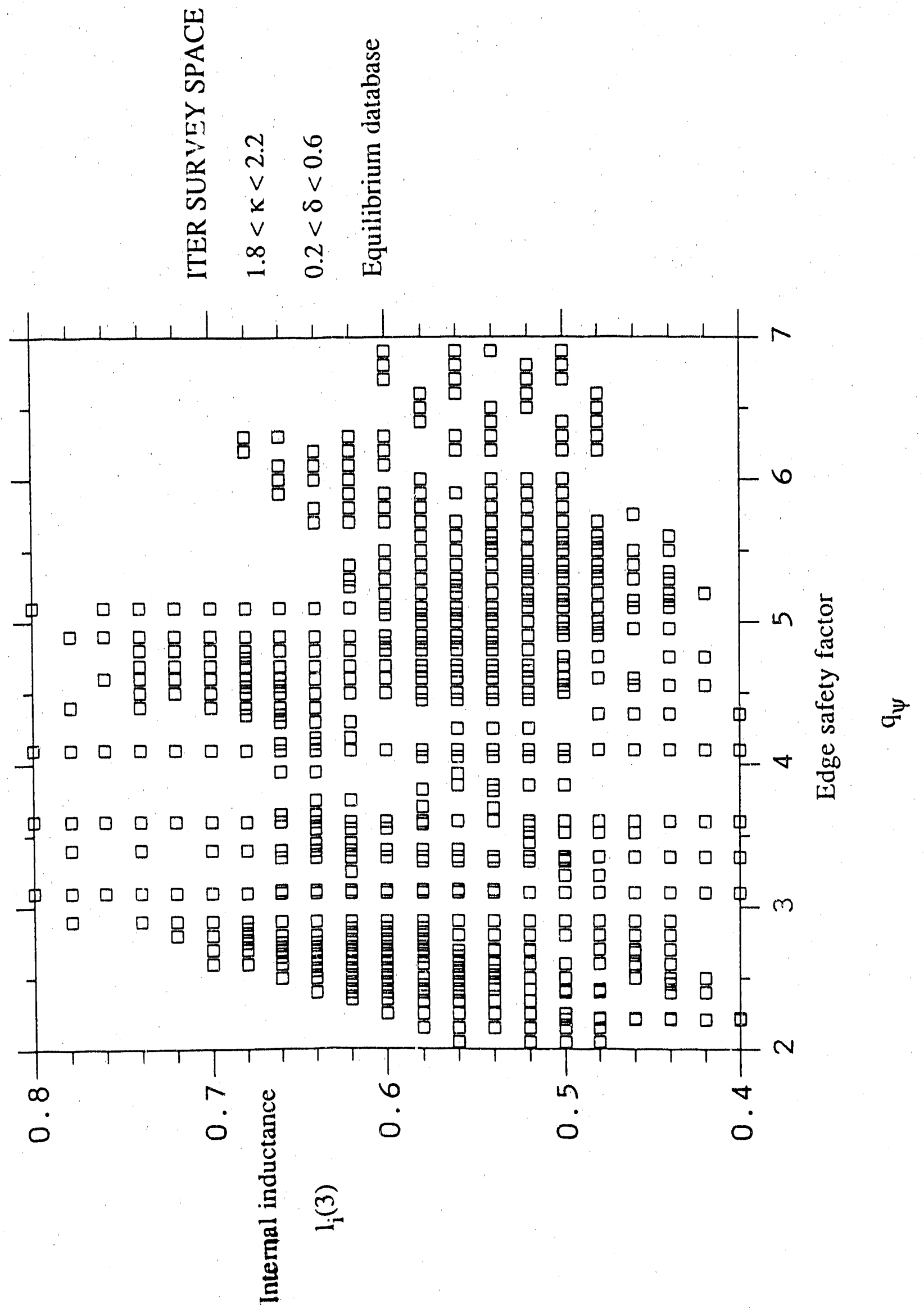

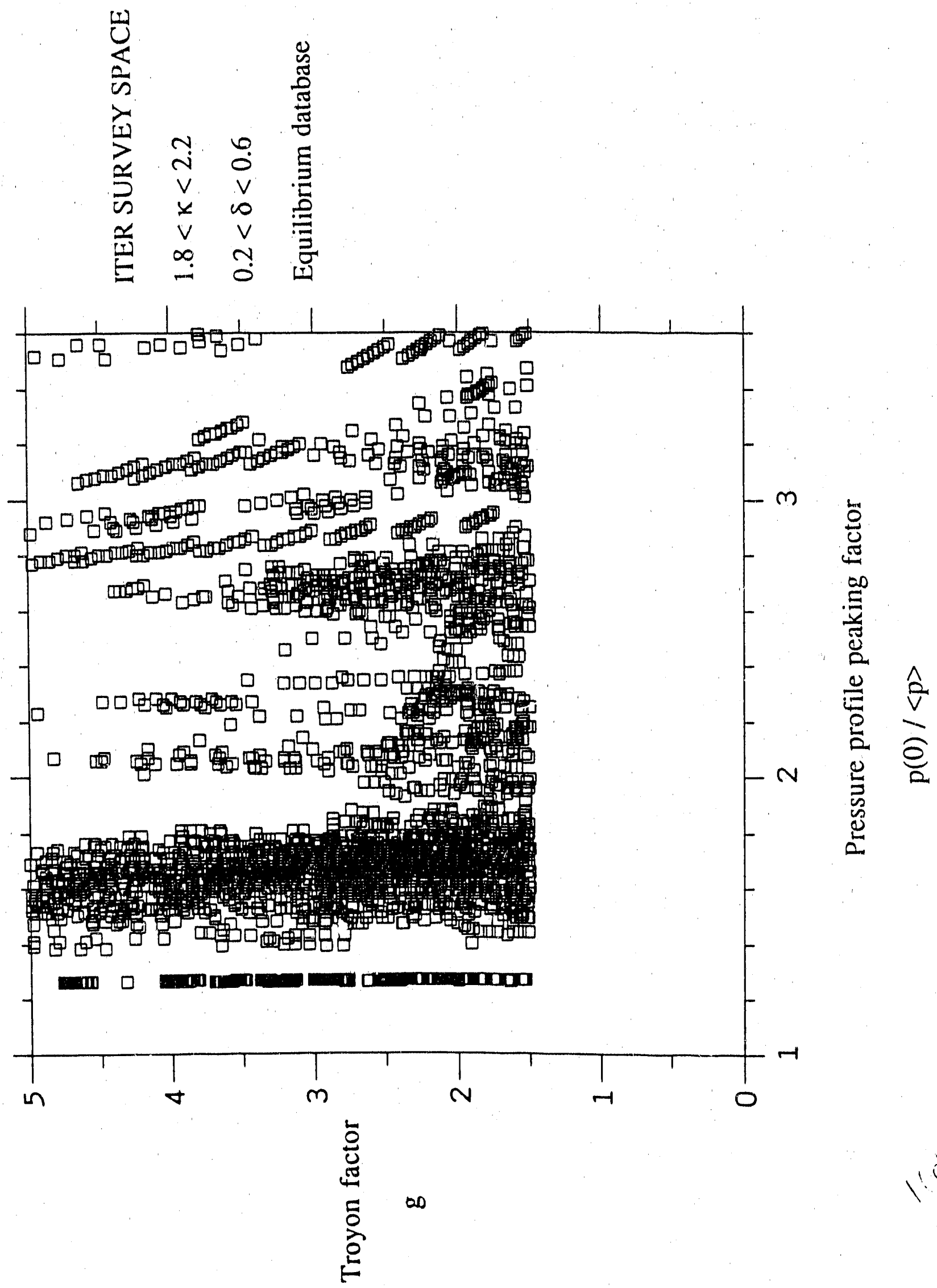


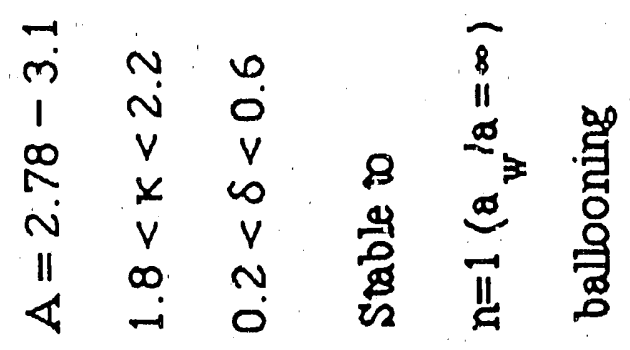

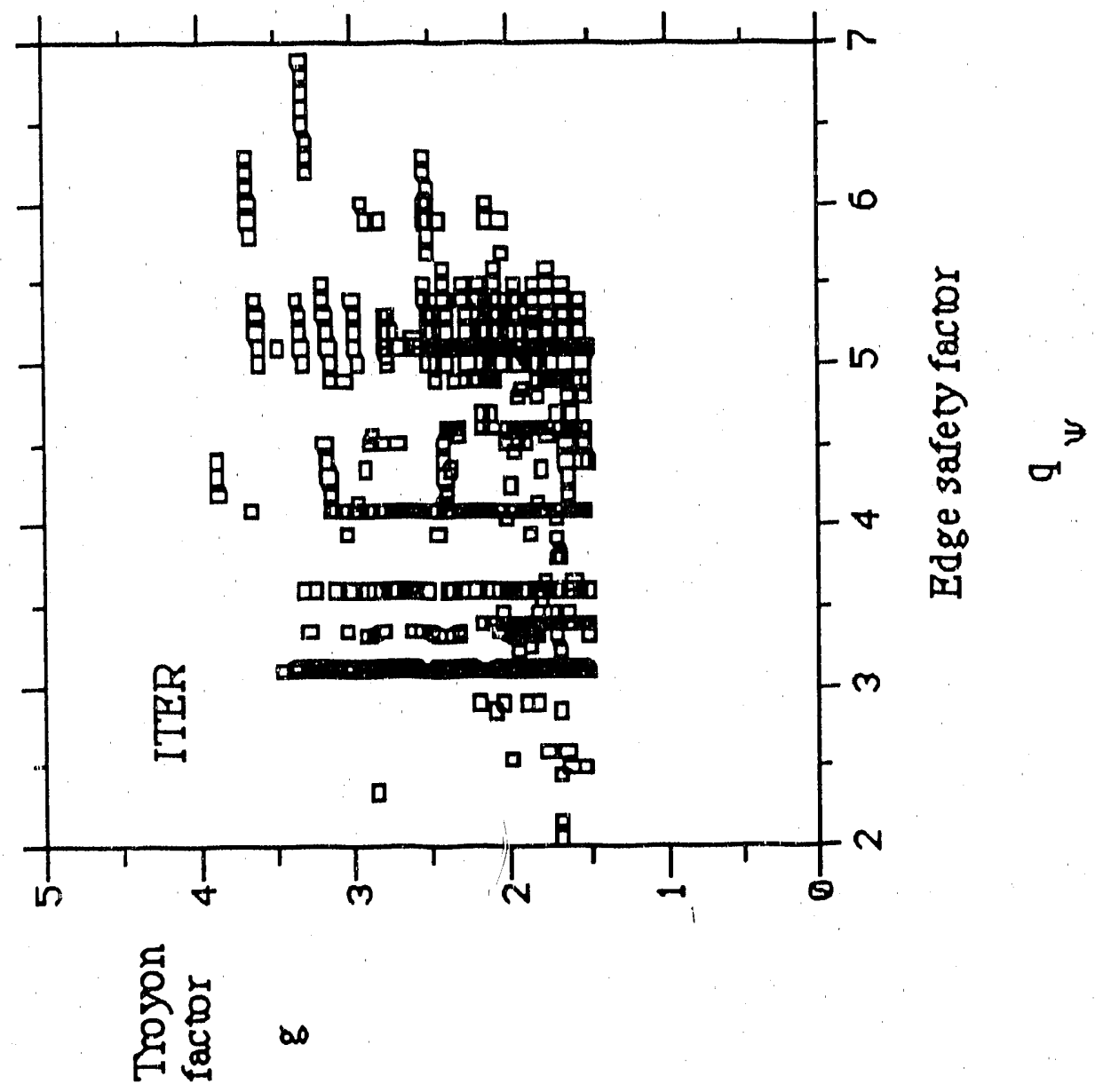



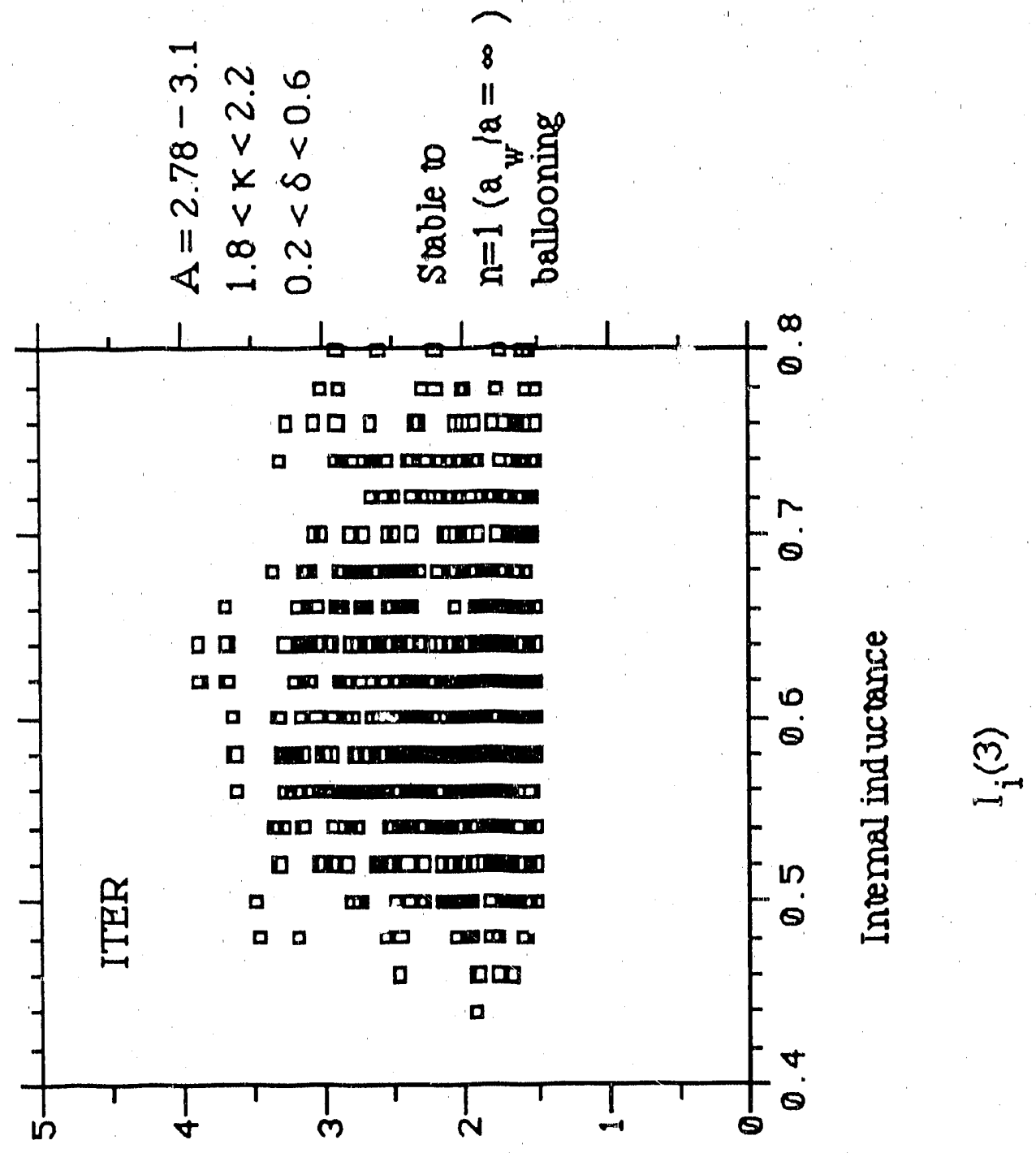

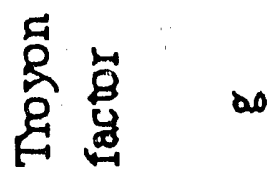




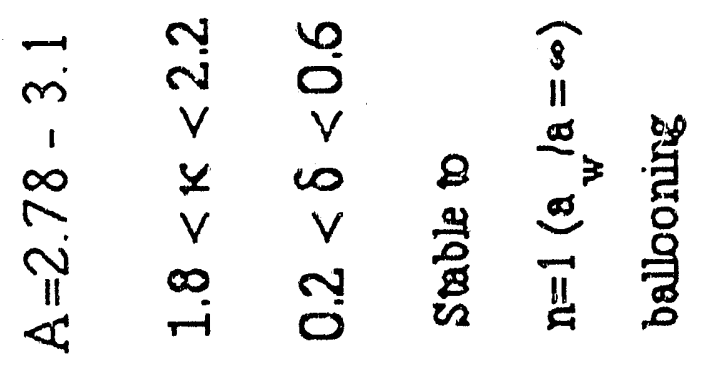

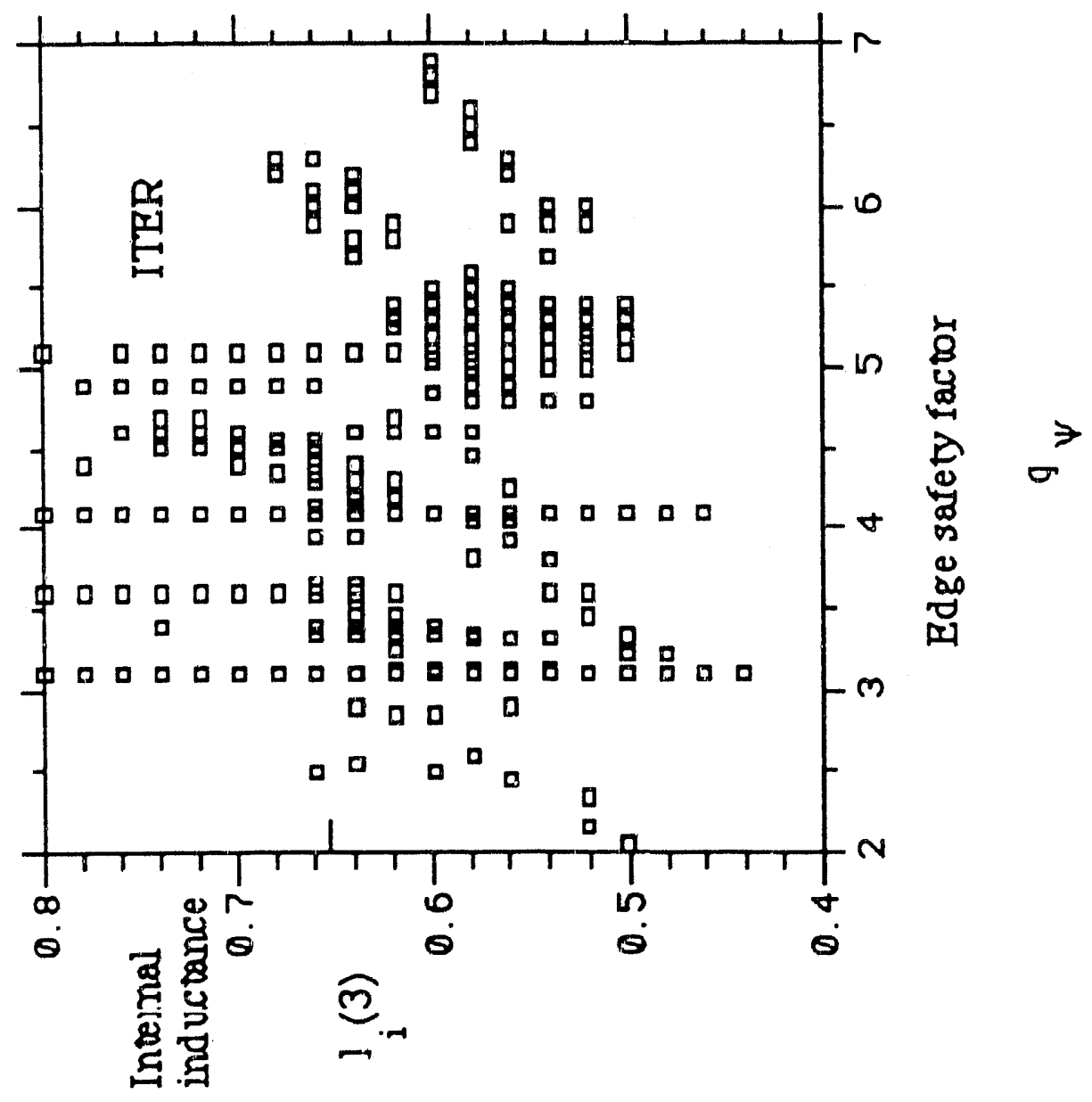




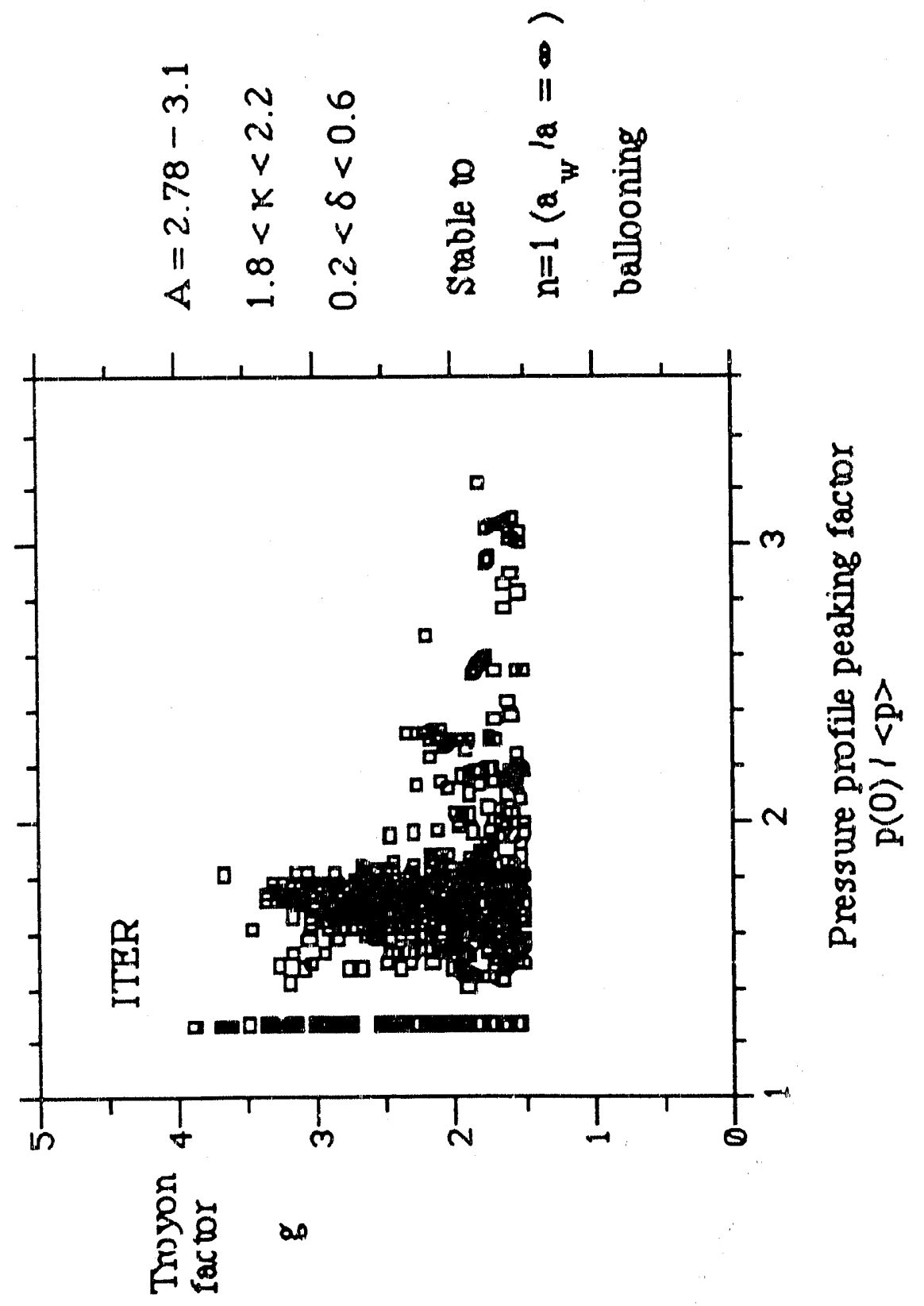




\section{$-6$}

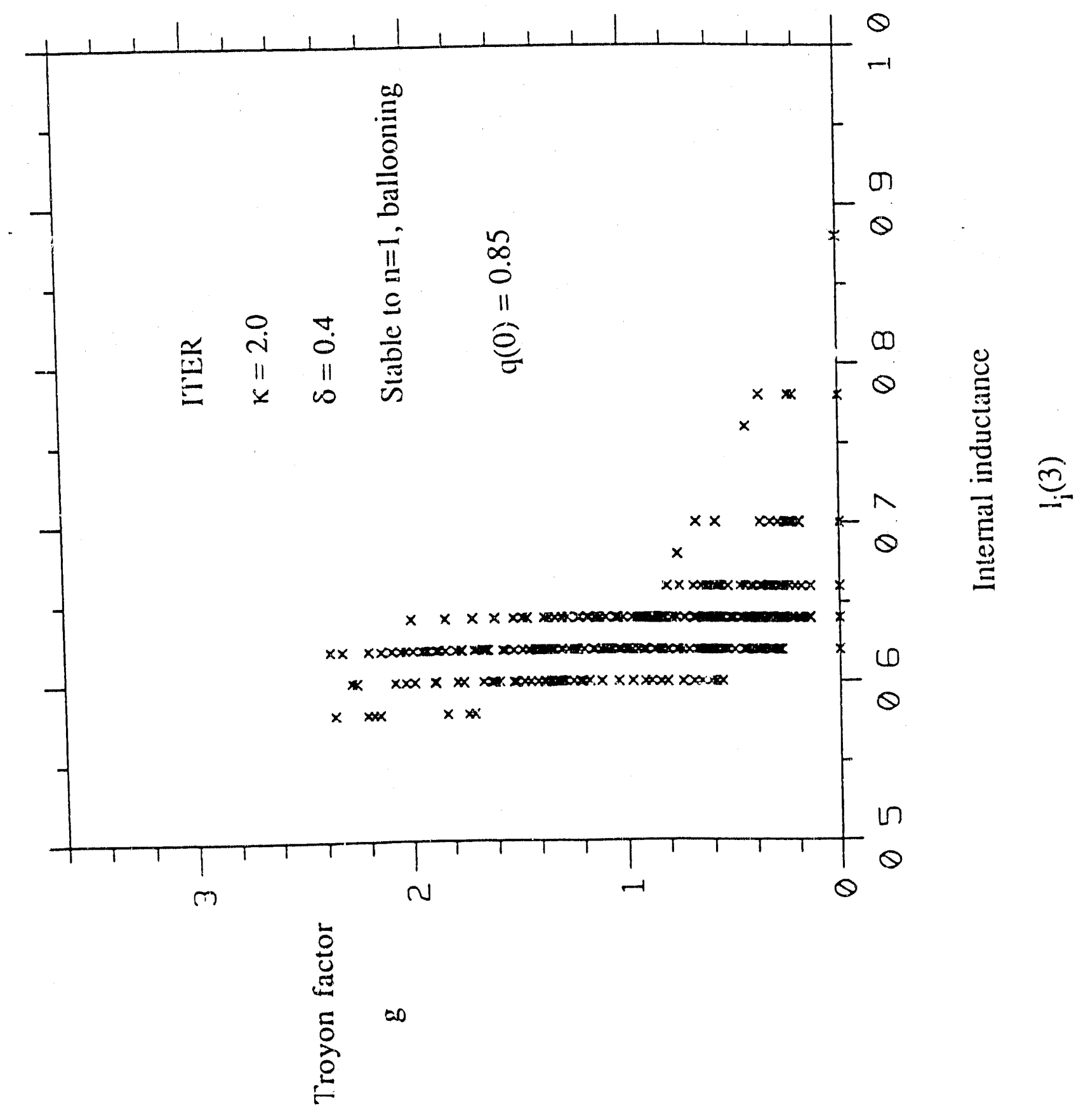


$\underset{5}{* 1}$

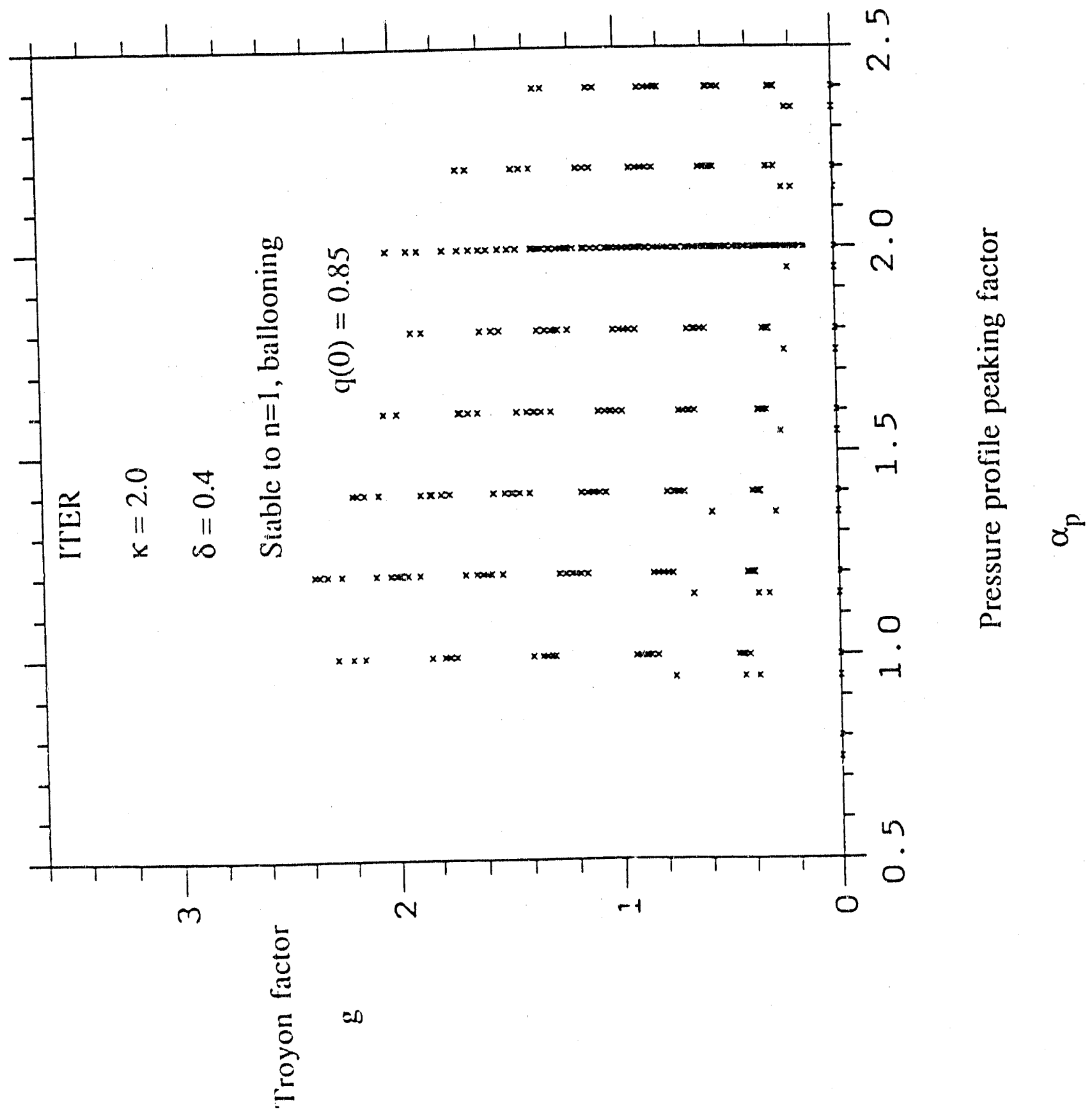



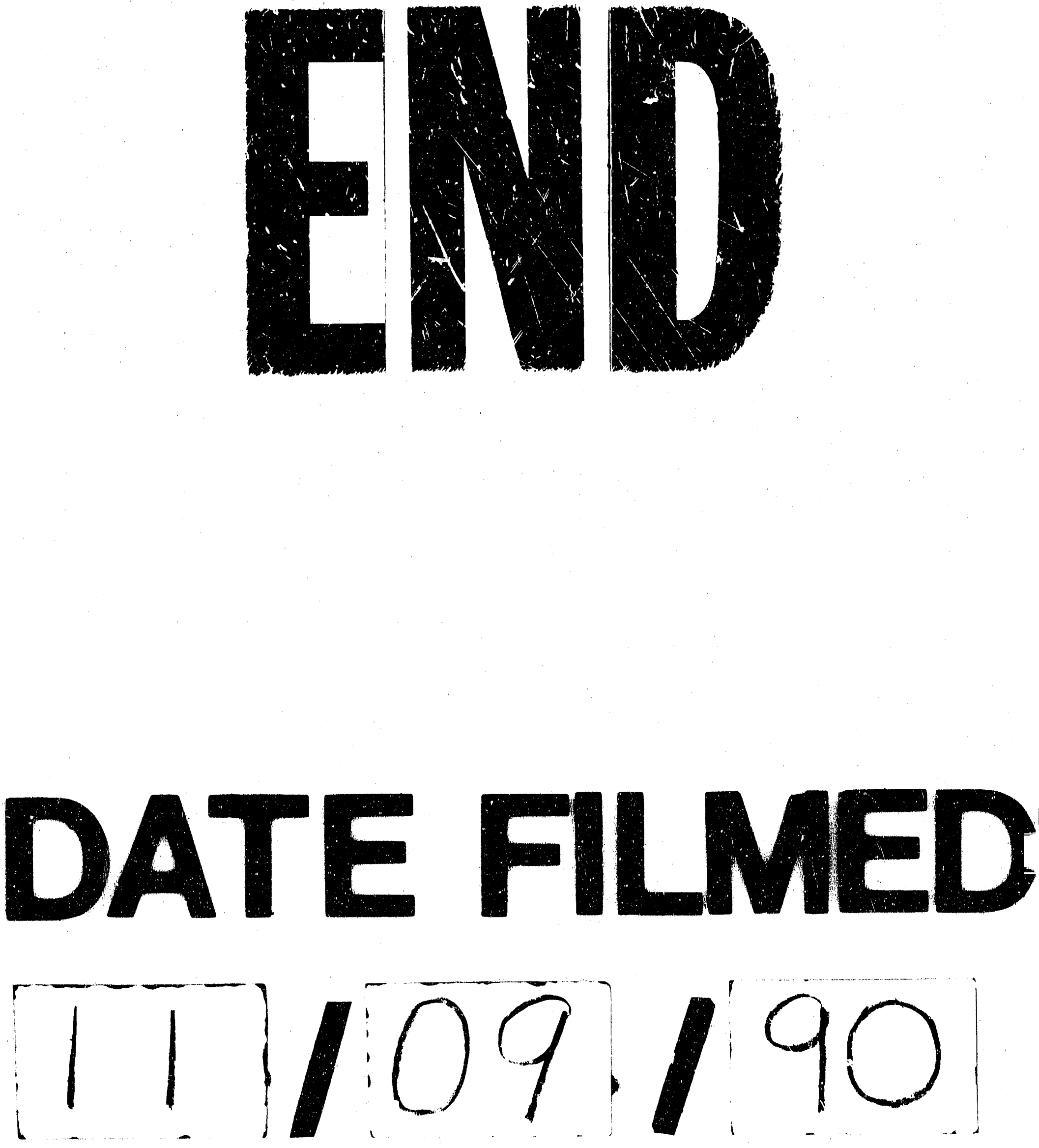
